\title{
The use of high-frequency ultrasound imaging and biofluorescence for in vivo evaluation of gene therapy vectors
}

\author{
Nicola Ingram ${ }^{1 *}$, Stuart A Macnab², Gemma Marston ${ }^{1}$, Nigel Scott ${ }^{3}$, lan M Carr ${ }^{1}$, Alexander F Markham', \\ Adrian Whitehouse ${ }^{2}$ and P Louise Coletta ${ }^{1}$
}

\begin{abstract}
Background: Non-invasive imaging of the biodistribution of novel therapeutics including gene therapy vectors in animal models is essential.

Methods: This study assessed the utility of high-frequency ultrasound (HF-US) combined with biofluoresence imaging (BFI) to determine the longitudinal impact of a Herpesvirus saimiri amplicon on human colorectal cancer xenograft growth.

Results: HF-US imaging of xenografts resulted in an accurate and informative xenograft volume in a longitudinal study. The volumes correlated better with final ex vivo volume than mechanical callipers $\left(R^{2}=0.7993, p=0.0002\right.$ vs. $\left.R^{2}=0.7867, p=0.0014\right)$. HF-US showed that the amplicon caused lobe formation. BFI demonstrated retention and expression of the amplicon in the xenografts and quantitation of the fluorescence levels also correlated with tumour volumes.
\end{abstract}

Conclusions: The use of multi-modal imaging provided useful and enhanced insights into the behaviour of gene therapy vectors in vivo in real-time. These relatively inexpensive technologies are easy to incorporate into pre-clinical studies.

Keywords: Biofluorescence, Ultrasound, Gene therapy, Imaging, Multi-modal, Colorectal cancer

\section{Background}

The use of non-invasive and accurate methods to determine tumour volume, as well as biodistribution and transduction imaging of novel therapeutics, is essential in experimental models in vivo. In particular, for gene therapy studies, knowledge of maintenance, expression and efficacy of the vector is a fundamental part of the testing process [1]. However, this is rarely achieved during the in vivo study of a novel gene therapy strategy, as often only longitudinal calliper measurements of xenograft growth or final histology after treatment are carried out. The spread or loss of a vector is rarely detected during the course of the experiment and for cancer treatment, not all therapies will result in a reduction in tumour volume.

\footnotetext{
* Correspondence: n.ingram@leeds.ac.uk

${ }^{1}$ School of Medicine, University of Leeds Brenner Building, St James's University Hospital, Leeds LS9 7TF, UK

Full list of author information is available at the end of the article
}

Therefore it is important to be able to examine the impact of a gene therapy vector during the in vivo testing phase using different assessment criteria, whilst being mindful of adhering to the principles of reduction, refinement and replacement in animal experiments.

Ultrasound is a non-invasive method that has been utilised recently for tumour growth studies in vivo and is used in the clinic for staging colorectal cancer among others [2,3]. High-frequency ultrasound (HF-US) machines are available for small animal imaging. They are relatively easy to use and give high resolution greyscale images of mouse anatomy [4]. They also give functional information on the vascular structure of xenografts through the use of contrast agents and are relatively inexpensive and portable compared to MRI machines [5]. Mechanical callipers, however, are still utilised extensively for therapeutic agent testing, especially in gene therapy applications on xenografts [6]. These are very cheap, non-invasive and allow multiple 
repeated measurements with no anaesthetic required. However, mechanical callipers assume that the growth of xenografts is always ellipsoid and can only measure growth above the skin surface of the animal. In addition, calliper measurements are also affected by skin thickness, subcutaneous fat layer thickness and compressibility of the tumour [7]. From our experience of xenograft growth in gene therapy and other therapeutic studies, we know that this ellipsoid growth pattern is rarely observed, especially as the tumour volume becomes large (above approximately $\left.300 \mathrm{~mm}^{3}\right)$.

A gene encoding a fluorescent or luminescent protein is often incorporated into gene therapy vectors in order to enumerate transduction efficiencies in vitro $[8,9]$. Moreover, these markers are also very useful for in vivo studies. Optical imaging chambers can be used to image the biodistribution of a vector when administered and can give an indication of the transduction efficiency in the target cells [10]. Optical imaging systems also allow the maintenance of a vector to be determined throughout the course of treatment, as well as examining the genetic stability of the vector over time. The first paper to prove that optical imaging could be used to measure tumour growth used bioluminescence of tumour cells in rat brain and was compared to MRI scans for tumour volume [11]. Imaging of stably-transfected cell lines containing red or green fluorescent protein (RFP or GFP) has been used to measure tumour and metastatic growth [12,13]. Recent work has also shown that fluorescent intensity correlates better with tumour volume than fluorescent area [14].

In the study described herein, we aimed to determine whether the use of HF-US measurements were more accurate than mechanical callipers in assessing xenograft volumes of tumour cells which were infected before injection with an experimental gene therapy vector. The use of HF-US to provide anatomical information on tumour growth and BFI to monitor expression of a gene therapy vector in longitudinal studies, were also analysed. The vector we used was a Herpesvirus saimiri (HVS) amplicon which contains the minimal elements for episomal maintenance without infectious capabilities $[9,15]$. This gamma-2 Herpesvirus amplicon can incorporate large amounts of heterologous DNA using a HVS-BAC (bacterial artificial chromosome) system and infects a broad range of human cells. The amplicon was previously stably transfected into the SW480 colorectal cancer cell line and contains a constitutively active GFP gene [16]. The presence of the GFP gene enabled monitoring of its persistence during xenograft growth in this study.

\section{Methods}

\section{Tumour model}

The colorectal cancer cell line, HCT116 was stablytransfected with an episomally-maintained Herpesvirus saimiri amplicon incorporating the GFP gene under the control of the Cytomegalovirus (CMV) promoter. These cells were grown in Dulbecco's Modified Eagle Medium (DMEM, Invitrogen) supplemented with $10 \%(\mathrm{v} / \mathrm{v})$ foetal calf serum, and $4 \mathrm{ul} / \mathrm{ml}$ Hygromycin B (Sigma, Poole U.K.) in $5 \% \mathrm{CO}_{2}$ at $37^{\circ} \mathrm{C}$ until there were enough cells for xenograft set up (approximately 3-4 weeks from infection). Parental cell lines were grown in DMEM and serum but no Hygromycin B. Two days before injection the amplicon-transfected cells were transferred to medium without any Hygromycin B.

$1 \times 10^{6}$ each of the parental and amplicon-containing cells were collected in 100ul of serum-free DMEM and injected subcutaneously into the right flank of 8-10 week old female CD1 nude mice to form xenografts. 6 mice per group were used. All experiments were performed following local ethical approval and in accordance with the Home Office Animal Scientific Procedures Act 1986.

\section{Tumour volume measurement with mechanical callipers}

Tumours were measured with mechanical callipers three times per week once the tumour became palpable (approximately 7-10 days following injection). Tumour volume was calculated as follows, unless otherwise stated: [17]

Tumor volume $=1 / 2$ (greatest longitudinal diameter $\times$ greatest transverse diameter ${ }^{2}$ )

After 40 days a final calliper measurement was taken, the xenografts were excised and weighed. If tumours exceeded the maximum permitted size of $17 \mathrm{~mm}$ diameter, the mice were sacrificed earlier. Mechanical calliper measurements were then taken in three dimensions ex vivo and the following tumour volume was calculated, unless otherwise stated:

$$
\text { Tumor volume }=\text { length } \times \text { height } \times(\pi / 6)
$$

\section{Anatomical imaging and tumour volume measurement using HF-US}

Once per week, mice were anaesthetised using 3\% (v/v) isofluorane and xenografts were imaged using a Vevo 770 high-frequency ultrasound machine (FUJIFILM VisualSonics, Inc, Toronto, Canada) equipped with a 40 $\mathrm{MHz}$ transducer. The focal depth of the transducer was placed at the mid-point of the centre of the tumour whilst scanning. A 3D scan of the tumour was then performed using the minimum step size possible for the length of tumour and regions of interest were drawn around the xenograft at approximately every 5 frames by an operator with extensive experience of HF-US and analysis [4]. A tumour volume was then calculated using 
the Vevo 770 version 3 software by creating a 3D reconstruction of these xenografts.

\section{Measurement of biofluorescence}

Before sacrifice at day 40, xenografts were imaged in an IVIS Spectrum (PerkinElmer, Inc, Massachusetts, USA). Standard settings for GFP were used (excitation 500nm and emission detected at $540 \mathrm{~nm}$ ) in epi-illumination at high intensity. Binning was set at 8 , field of view was $13.1 \mathrm{~cm}$ and $\mathrm{f}$ stop was 2 . Regions of interest of the same size were drawn around each xenograft and the total radiant efficiency ([photons $\left./ \mathrm{s}] /\left[\mu \mathrm{W} / \mathrm{cm}^{2}\right]\right)$ was calculated within this using Living Image version 4.2 software (PerkinElmer, Inc, Massachusetts, USA).

\section{Histology and morphology of xenografts}

Once the xenographs were excised, photographs were taken of the intact tumours. The tumours were then cut in half and fixed in $4 \%(\mathrm{w} / \mathrm{v})$ paraformaldehyde in PBS overnight. After processing and embedding in wax, sections were dewaxed, rehydrated and stained with haematoxylin and eosin. Sections were assessed by an experienced histopathologist.

\section{Statistical analysis}

Analysis of the tumour volumes and vector expression obtained by these methods used Pearson correlations. Positive correlations produced a positive $\mathrm{R}^{2}$ value and were considered significant if $\mathrm{p}<0.05$. Agreement between the methods was then further analysed by Bland-Altman plots where the central line (mean of differences or bias) and 2 standard deviation (SD) limits of agreement were generated. The bias was considered significant if 0 was not included within these standard deviation lines. These calculations were carried out using GraphPad Prism version 5 (GraphPad Software, Inc, La Jolla, California, USA).

\section{Results}

Comparison of tumour growth curves generated using mechanical callipers or HF-US

HF-US was used to determine the tumour volume during the growth course of the xenografts derived from the parental cell line and amplicon-infected cell line and compared to the volume calculated from mechanical calliper measurements. The tumour volumes generated from the two methods are shown in Figure 1. The ampliconinfected xenograft tumours grew more slowly than the parental cells and this was detected by both measurement methods. Tumour volumes by HF-US generated smaller calculated tumour volumes than those using mechanical callipers. At day 28 for example, calliper assessed xenograft tumour volumes were calculated to be more than twice the volumes generated using HF-US imaging. This difference was even greater for the amplicon-infected xenografts as these were 3.3 times larger when measured using mechanical callipers compared to HF-US.

\section{Comparison of tumour volume measurement methods to the volume calculated using ex vivo calliper} measurements

HF-US measurements correlated more closely than mechanical callipers (denoted as in vivo callipers on the graphs) to the final ex vivo calliper measurement at the end of the period of xenograft growth which is our most accurate measurement (Figure $2 \mathrm{a}$ and b). Thus the tumour growth curves in Figure 1 are an over-estimation if mechanical callipers are used compared to HF-US measurements. Alternative formulae for tumour volume calculation for both in vivo and ex vivo calliper measurements were examined and compared to HF-US (Table 1) [17]. As before, HF-US measurements correlated more closely to either ex vivo volume formula than any in vivo volume formula and no difference in correlation was

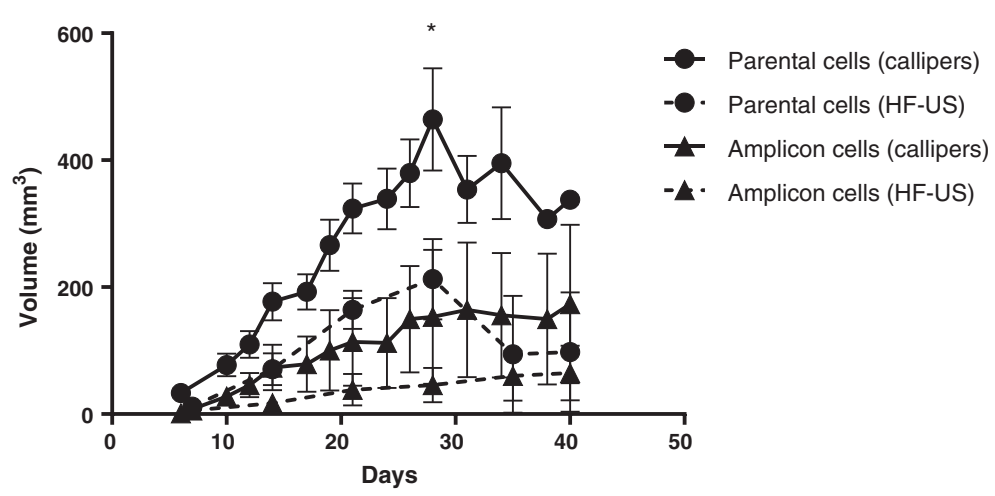

Figure 1 Longitudinal growth of xenograft tumours using mechanical callipers and HF-US. Growth of xenografts generated from each line using mechanical Vernier callipers on the external surface of the animal (in vivo calliper volume - solid lines) and using 3D high-frequency ultrasound scans and calculating volumes by drawing regions of interest on each frame (dotted lines). Mean volume $+/$ - standard deviation of each group is shown ( $n=5$ for calliper measurements and 6 for HF-US) * denotes that mice were culled in this group after this point due to large tumour volumes ( $n=2$ from day 28$)$. 
(a)

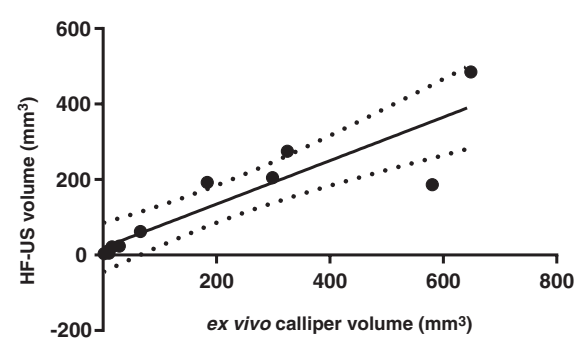

(b)

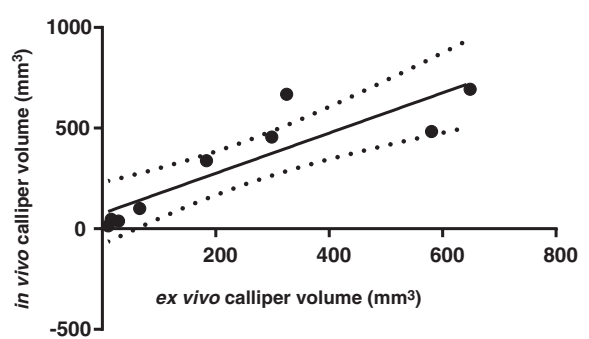

Figure $2 \mathrm{HF}$-US correlates more closely to the ex vivo tumour volume than using mechanical callipers in vivo. The tumour volume generated by HF-US correlates more closely with the final ex vivo calliper volume than the in vivo calliper volume. (a) The Pearson correlation plot of HF-US volumes versus ex vivo volumes has a higher $R^{2}$ value $\left(R^{2}=0.7993,95 \% \mathrm{Cl}=0.6342-0.9724, p=0.0002\right.$, two-tailed) than in vivo calliper volumes versus ex vivo volumes (b) $\left(R^{2}=0.7867,95 \% \mathrm{Cl}=0.5421-0.9761, p=0.0014\right.$, two-tailed). The solid line denotes line of best fit and dotted lines indicate the $95 \%$ confidence band, $n=10$.

found between the two ex vivo volume formulae and HFUS volumes. Using the formula $\pi / 6 \times(\mathrm{L} \times \mathrm{W})^{3 / 2}$ for in vivo calliper volumes gave a higher correlation to both HF-US volumes and to mass of tumour than the other two equations.

\section{Comparison of tumour volume measurement methods to} final tumour mass

After sacrifice, the resulting xenograft tumours were excised and weighed. Using Pearson correlation coefficients and linear regression analysis, final in vivo calliper measurements had a lower correlation coefficient to tumour mass than HF-US. The tumour volumes calculated from ex vivo calliper measurements of the excised xenograft had the highest correlation coefficient to tumour mass (Figure 3 $\mathrm{a}, \mathrm{b}$ and $\mathrm{c}$ and Table 1). Bland-Altman graphs show a smaller 95\% confidence interval between HF-US volumes and the ex vivo calliper measurement compared to the confidence interval between final in vivo calliper and the ex vivo calliper measurements (Figure $4 \mathrm{a}$ and $\mathrm{b}$ ). This demonstrates a smaller difference between HF-US and the ex vivo calliper measurement methods than between in vivo and ex vivo callipers.

\section{HF-US imaging and BFI of tumour anatomy and gene therapy vector expression}

In addition to HF-US, the use of BFI allowed the persistence and expression of the amplicon to be tracked in vivo. The HF-US images and photographs show that the amplicon-containing xenografts grew in distinct lobes

Table 1 Pearson correlation coefficients of xenograft tumour volumes using different ellipsoid formulae and measured using mechanical callipers, HF-US or mass

\begin{tabular}{|c|c|c|c|c|c|}
\hline & & $\pi / 6 \times \mathrm{L} \times \mathrm{W} \times \mathrm{H}$ & $0.5 \times \mathrm{L} \times \mathrm{W} \times \mathrm{H}$ & HF-US & Mass (g) \\
\hline \multirow[t]{3}{*}{$0.5 \times L \times W^{2}$} & $\mathrm{R}^{2}$ & 0.7867 & 0.7867 & 0.8576 & 0.7843 \\
\hline & $95 \% \mathrm{Cl}$ & $0.5421-0.9761$ & $0.5421-0.9761$ & $0.7110-0.9827$ & $0.4811-0.9792$ \\
\hline & $\mathbf{p}$ & 0.0014 & 0.0014 & 0.0001 & 0.0034 \\
\hline \multirow[t]{3}{*}{$\pi / 6 \times L \times W^{2}$} & $\mathrm{R}^{2}$ & 0.7867 & 0.7867 & 0.8576 & 0.7843 \\
\hline & $95 \% \mathrm{Cl}$ & $0.5421-0.9761$ & $0.5421-0.9761$ & $0.7110-0.9827$ & $0.4811-0.9792$ \\
\hline & $\mathrm{p}$ & 0.0014 & 0.0014 & 0.0001 & 0.0034 \\
\hline \multirow[t]{3}{*}{$\pi / 6 \times(L \times W)^{3 / 2}$} & $R^{2}$ & 0.8325 & 0.8325 & 0.8636 & 0.8492 \\
\hline & $95 \% \mathrm{Cl}$ & $0.6300-0.9817$ & $0.6300-0.9817$ & $0.7223-0.9835$ & $0.6184-0.9860$ \\
\hline & p & 0.0006 & 0.0006 & 0.0001 & 0.0011 \\
\hline \multirow[t]{3}{*}{ HF-US } & $\mathrm{R}^{2}$ & 0.7993 & 0.7993 & & 0.8470 \\
\hline & $95 \% \mathrm{Cl}$ & $0.6342-0.9724$ & $0.6342-0.9724$ & & $0.6135-0.9857$ \\
\hline & $\mathbf{p}$ & 0.0002 & 0.0002 & & 0.0012 \\
\hline \multirow[t]{3}{*}{ Mass (g) } & $\mathrm{R}^{2}$ & 0.9254 & 0.9254 & & \\
\hline & $95 \% \mathrm{Cl}$ & $0.7580-0.9946$ & $0.7580-0.9946$ & & \\
\hline & $p$ & 0.0005 & 0.0005 & & \\
\hline
\end{tabular}

Three different formulae for generating in vivo tumour volumes using callipers are shown to the left and two different formulae for ex vivo tumour volumes using callipers on top. Each have been subject to pairwise comparison to determine the different correlation coefficients. 
(a)

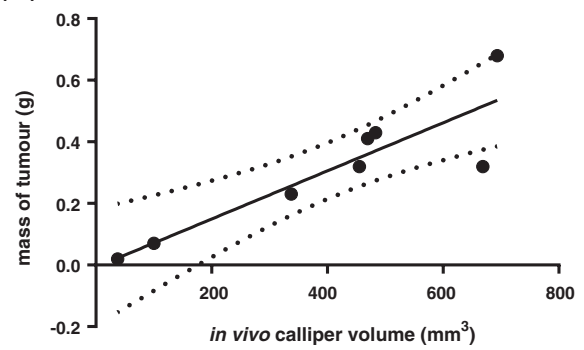

(c)

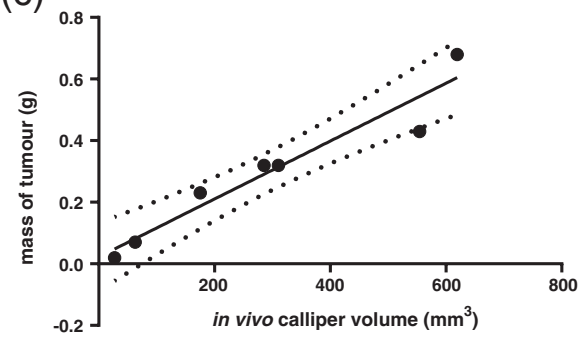

(b)

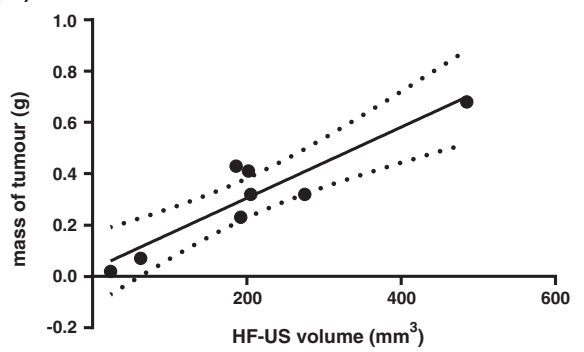
volume. Pearson correlations showed that in vivo calliper volumes correlated the least with tumour mass (Figure $3 \mathbf{a}, R^{2}=0.7843$,

$95 \% \mathrm{Cl}=0.4811-0.9792, p=0.0034$ ), followed by HF-US volume (Figure $3 \mathbf{b}, R^{2}=0.8470,95 \% \mathrm{Cl}=0.6135-0.9857, \mathrm{p}=0.0012$ ) whereas ex vivo calliper volume showed the best correlation (Figure $3 \mathbf{c}, \mathrm{R}^{2}=0.9254,95 \% \mathrm{Cl}=0.7580-0.9946, \mathrm{p}=0.0005$ ). Smaller tumours were not accurately weighed by the balance therefore $n=8$.

unlike the parental cell xenografts. These distinct lobes were visible even from day 8 on the HF-US images in comparison to the parental cell xenografts, thus allowing very early detection of anatomic differences between the two groups in vivo which was not possible to elucidate from calliper measurements alone. The detailed greyscale anatomical images using HF-US showed both lighter and darker areas (derived from areas that are more or less echogenic to ultrasound) (Figure 5). The relatively lighter areas within the xenograft were not adipose tissue and corresponded to denser tumour tissue and from histology we observed that the darker areas are necrotic tissue and when the tumours were excised open, a liquid interior core was found (Figure 6a). Amplicon infection of the cells caused formation of syncitia (fused cells) during xenograft growth, which was not evident in the parental (a)

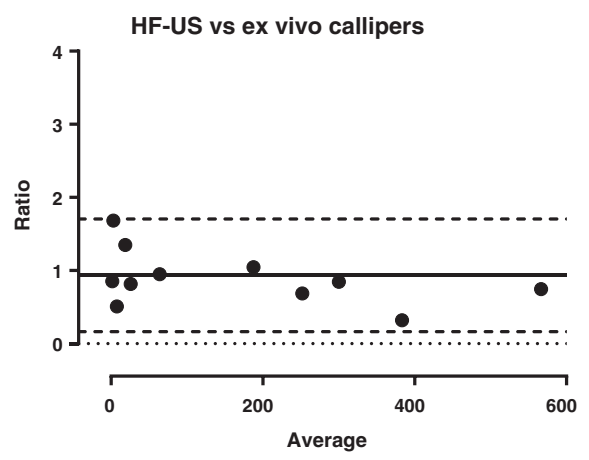

(b)

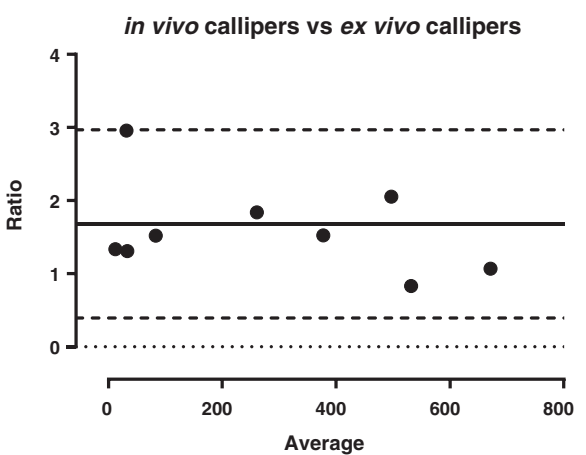

Figure 4 Bias assessment of each method for tumour volume calculation. HF-US volumes show less bias than in vivo calliper volumes when compared to ex vivo calliper volumes. The ratios of HF-US to ex vivo calliper volumes ( $y$-axis) were compared to the average value of the measurements (x-axis). Bland-Altman plots were generated comparing the bias between HF-US and ex vivo calliper volumes (a) and in vivo calliper volumes compared to ex vivo calliper volumes (b). The solid line denotes the bias (the average of the differences between the two measurement methods) and the dashed lines define the $95 \%$ confidence limits. The dotted line defines zero. HF-US could detect much smaller tumour volumes than callipers therefore $n=11$ in (a) and $n=10$ in (b). 


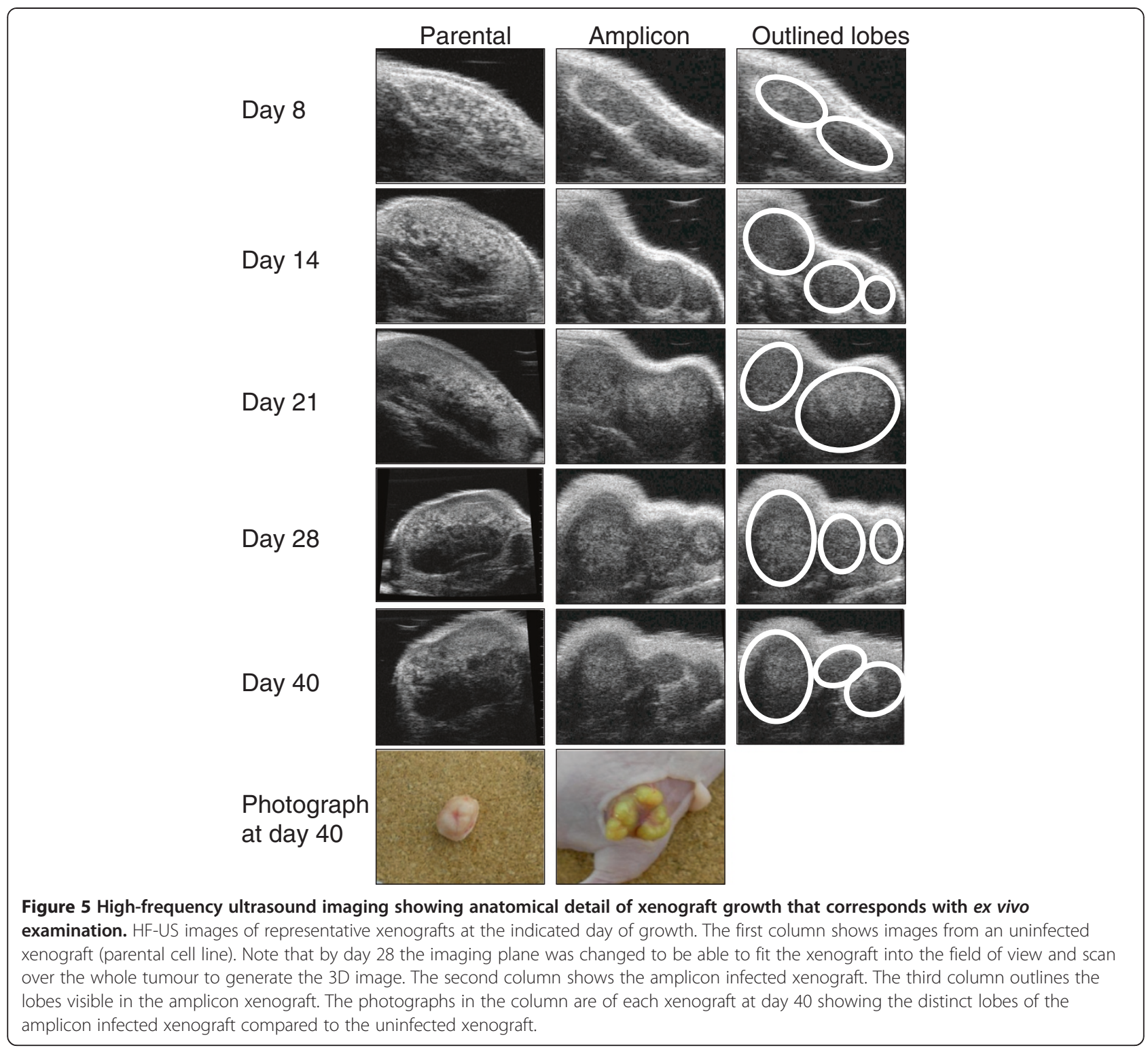

cell xenografts, as shown in Figure 6b. The presence of lobes seen by HF-US can also be discerned in the fluorescent image taken by the IVIS Spectrum instrument (Figure 6c).

\section{Correlation of total radiant efficiency (fluorescence) and tumour volume measurements}

The measurement of levels of fluorescence was determined for the amplicon-infected xenografts using an IVIS Spectrum and the Living Image software and plotted alongside the ex vivo calliper volume (Figure 7a). These measurements show a similar pattern for the amplicon cell line in terms of fluorescence emission and calliperderived tumour volume. In vivo calliper measurements on the final day of growth were less significantly correlated to fluorescence measurements than calliper measurements of the ex vivo xenografts (in vivo callipers, $\mathrm{R}^{2}=0.8882$, 95\% CI $=0.3568-0.9963, \mathrm{p}=0.0164$ compared with ex vivo callipers, $\mathrm{R}^{2}=0.941795 \% \mathrm{CI}=0.5518-0.9938, \mathrm{p}=0.0050$ ) (Figure $7 \mathrm{~b}$ and $\mathrm{c}$ ). HF-US volume measurements had a better correlation coefficient to fluorescence measurements than the ex vivo calliper measurements $\left(\mathrm{R}^{2}=\right.$ 0.8895, 95\% CI =0.5606-0.9939, $\mathrm{p}=0.0048$ ) (Figure $7 \mathrm{~d}$ ). However, it must be noted these are based on small numbers in each group, as only the amplicon-infected cells contained GFP and not the parental cells.

\section{Discussion}

Multimodal imaging in gene therapy applications is a useful tool to shed light on the behaviour of vectors during in vivo testing. In this study, the use of HF-US imaging identified anatomical differences during growth 


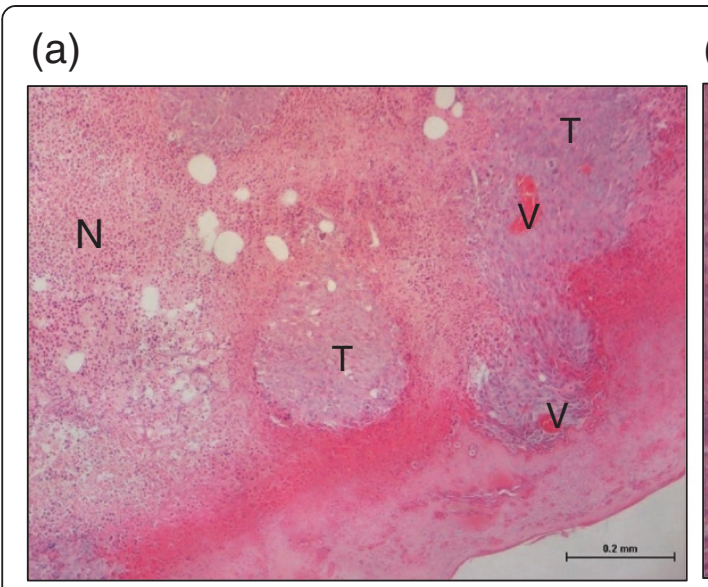

(b)

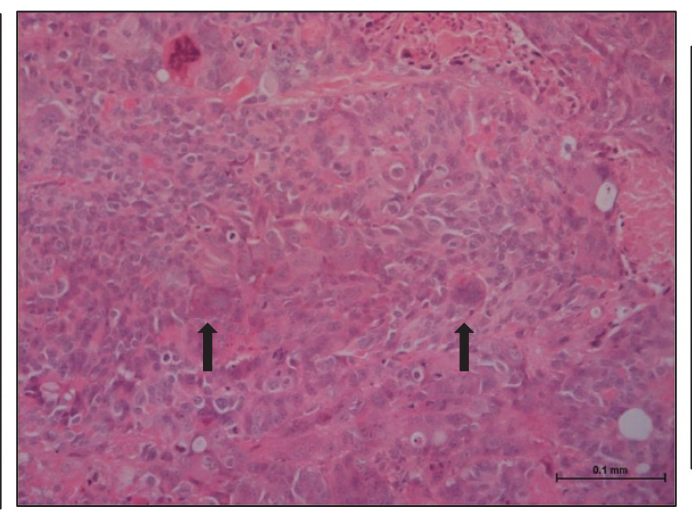

(c)

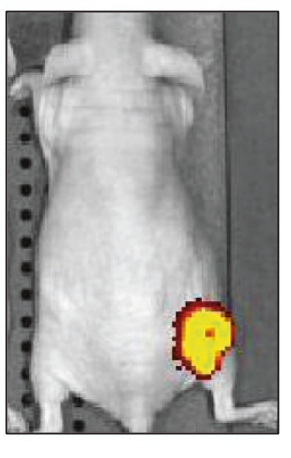

Figure 6 Histological examination of the tumours. Haematoxylin and Eosin stained sections of xenografts. (a) Parental cell xenografts typically showed islands of viable tumour ( $T$ ) containing blood vessels $(V)$ with large areas of necrosis (N). (b) amplicon infected xenografts show syncitia (arrowed) present amongst the tumour cells, which were not observed in any of the parental cell line xenografts. (c) The IVIS Spectrum image clearly shows the fluorescence emission from the xenograft lobes in an amplicon-infected xenograft, pseudo-coloured with the software default settings of red to yellow for increasing intensity of signal (parental cell line xenografts contained no GFP and showed no signal by BFI).
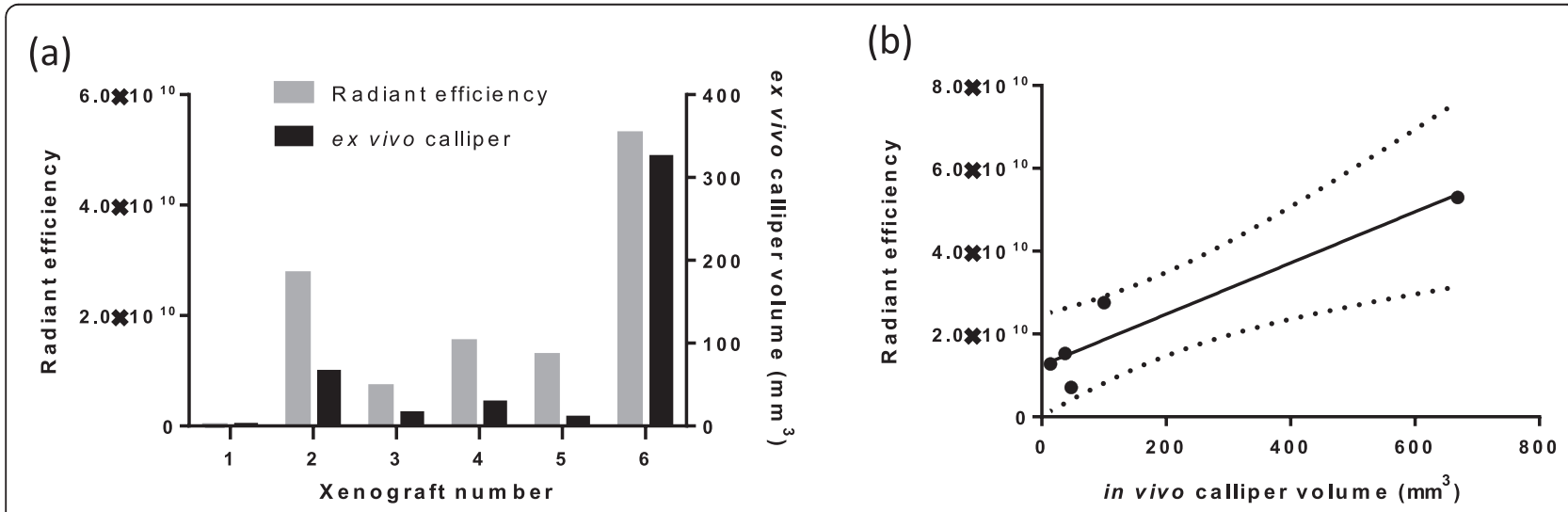

(c)

(d)
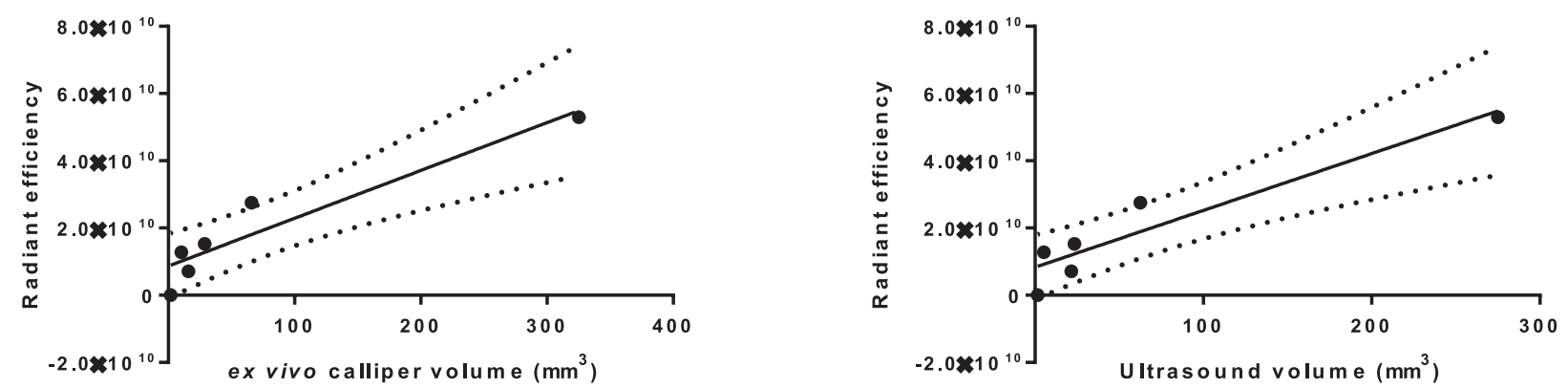

Figure 7 Total radiant efficiency correlates with xenograft tumour volume. (a) The total radiant efficiency of each amplicon-infected xenografts is plotted on the left $y$-axis of the graph alongside the ex vivo calliper tumour volume which is plotted on the right $y$-axis of the graph. Total radiant efficiency compared to in vivo calliper volume is shown (b), $R^{2}=0.8882,95 \% \mathrm{Cl}=0.3568-0.9963, p=0.0164$ ( $n=5$ as one xenograft was too small to be measured by callipers in vivo). Total radiant efficiency compared to ex vivo calliper volumes is shown in (c), $R^{2}=0.941795 \%$ $\mathrm{Cl}=0.5518-0.9938, \mathrm{p}=0.0050, \mathrm{n}=6$. Total radiant efficiency compared to HF-US volumes is shown in $(\mathbf{d}), \mathrm{R}^{2}=0.8895,95 \% \mathrm{Cl}=0.5606-0.9939$, $p=0.0048, n=6$. 
between the parental cell line and the vector-transfected cell line in a xenograft model, even from day 8 after implantation. It has been shown that HF-US can more accurately measure tumour volume compared to the traditional mechanical callipers, as demonstrated in this paper and by others $[2,18]$. The use of different ellipsoid volume formulae to generate the tumour volumes from calliper measurements made small differences in accuracy where the highest correlation to mass was found using $\pi / 6 \times(\mathrm{L} \times \mathrm{W})^{3 / 2}$ rather than the more commonly used $0.5 \times \mathrm{L} \times \mathrm{W}^{2}$ as described previously (although based on only one paper [17]). Correlation to determining volume by water displacement would be the gold standard and would be a useful addition to this study. HF-US volume generation and mechanical calliper measurements by multiple operators would also be valuable for determining variability as these measurements are subject to bias from operators. Jensen and colleagues compared volumes determined by microCT, ${ }^{18}$ F-FDG-microPET and external callipers, to an ex vivo reference volume calculated by weight and density [19]. They demonstrated that micro-CT was more accurate and reproducible between observers than either external callipers or ${ }^{18}$ F-FDG-microPET. They also showed that ${ }^{18}$ F-FDGmicroPET was not so useful for determining tumour size, although there was some correlation $\left(R^{2}=0.75\right)$. This was similar to our findings with biofluorescence imaging. As with our study, this functional tumour imaging modality is useful for metabolic imaging and should give an indication of the effect of a gene therapy vector on tumour viability. In the current study, HF-US accurately showed the slower tumour growth of the vectortransfected cell line compared to the parental cell line, as predicted from in vitro cell growth curves [16]. However, lobe formation was unexpected. We are currently investigating whether this is due to the GFP gene or other components of the vector backbone. We also demonstrated the utility of the different greyscale textures in monitoring different patterns of growth. The discrimination of areas of necrosis and high vascularity (using contrast agents) was also possible. This should allow real-time monitoring of agents that currently have little apparent effect on tumour volume but may have useful effects of antiangiogenesis or inducing cell senescence. HF-US would be of particular use for very small xenografts, orthotopic models to in transgenic mice such as the $\mathrm{Apc}{ }^{\mathrm{Min} /+}$ mouse, where callipers cannot access the tumour. Indeed, gene therapy vectors are also used in non-cancer applications such as diabetes or organ regeneration, where callipers may not be used to measure disease progress or regression. In these cases, HF-US would be invaluable in monitoring progress longitudinally without sacrifice of mice.

In addition to HF-US images, the use of biofluorescence allowed monitoring of tumour growth patterns and cor- related well with final tumour volumes (although it must be noted this was based on small numbers with a wide variation). This technique is a simple and very quick method of visualising the tumour and much less expensive than ${ }^{18} \mathrm{~F}$-FDG-microPET, for example. Bio-fluorescence is also applicable to patients. It is currently being trialled in surgery on human tumours to define tumour margins for resection [20]. The monitoring of these two cell lines grown as xenografts showed that the presence and expression of the vector was maintained within the tumour over the duration of the experiment. This information is of great value for gene therapy applications as silencing of the vector can occur, which may not be evident from growth curves or even from immunohistochemistry on ex vivo tumour sections for vector proteins. Linkage of the therapeutic gene of interest to a fluorescent marker gene via an IRES (internal ribosomal entry site) sequence or as a fusion protein would yield valuable information on the efficacy of expression during the time course of an in vivo experiment. It may also be used to reduce costs by eliminating animals in which the introduction of a vector by injection has not been successful.

\section{Conclusions}

In conclusion we believe that multi-modal imaging provides useful and enhanced insights into the behaviour of gene therapy vectors in vivo. Addition of imaging to gene therapy protocols would be straightforward especially in the case of relatively inexpensive ultrasound and biofluorescence imaging. The use of multi-modal imaging can give important information on the behaviour of gene therapy vectors in real-time, rather than traditional calliper measurements and final histological examination.

\section{Abbreviations \\ HF-US: High-frequency ultrasound; BFl: Biofluorescent imaging; HVS: Herpesvirus saimiri; GFP: Green fluorescent protein.}

\section{Competing interests}

The authors declare no competing interests financial or otherwise.

\section{Authors' contributions}

$\mathrm{Nl}$ carried out the experiments, analysed the data and wrote the manuscript. SAM generated the stably-infected cell line. GM imaged xenografts by HF-US and generated tumour volumes. IMC generated the amplicon. NS provided histological information on the resulting xenografts. AFM provided discussion of the results. AW was involved in study design, discussion of results and generation of the amplicon. PLC was involved in study design, discussion of results and manuscript editing. All authors read and approved the final manuscript.

\section{Acknowledgements}

This study was funded by EPSRC grant number EP/I000623/1 and YCR grant number L332.

\section{Author details}

${ }^{1}$ School of Medicine, University of Leeds Brenner Building, St James's University Hospital, Leeds LS9 7TF, UK. ${ }^{2}$ School of Molecular and Cellular Biology, Faculty of Biological Sciences and Astbury Centre for Structural 
Molecular Biology, University of Leeds, Leeds LS2 9JT, UK. ${ }^{3}$ Department of Histopathology, Bexley Wing, St James's University Hospital, Leeds LS9 7TF, UK.

Received: 1 February 2013 Accepted: 1 November 2013

Published: 12 November 2013

\section{References}

1. Waerzeggers $Y$, et al: Methods to monitor gene therapy with molecular imaging. Methods 2009, 48(2):146-160.

2. Cheung AM, et al: Three-dimensional ultrasound biomicroscopy for xenograft growth analysis. Ultrasound Med Biol 2005, 31(6):865-870.

3. Samee A, Selvasekar CR: Current trends in staging rectal cancer. World J Gastroenterol 2011, 17(7):828-834.

4. Abdelrahman MA, et al: High-Frequency Ultrasound for In Vivo Measurement of Colon Wall Thickness in Mice. Ultrasound Med Biol 2012, 38(3):432-442.

5. Lee DJ, et al: Relationship between retention of a vascular endothelial growth factor receptor 2 (VEGFR2)-targeted ultrasonographic contrast agent and the level of VEGFR2 expression in an in vivo breast cancer model. J Ultrasound Med 2008, 27(6):855-866.

6. Zhao $Y$, et al: Increased antitumor capability of fiber-modified adenoviral vector armed with TRAIL against bladder cancers. Mol Cell Biochem 2011, 353(1-2):93-99.

7. Euhus DM, et al: Tumor measurement in the nude mouse. J Surg Oncol 1986, 31(4):229-234.

8. Sims $K$, et al: In vitro evaluation of a 'stealth' adenoviral vector for targeted gene delivery to adult mammalian neurones. J Gene Med 2009, 11(4):335-344

9. Smith $P G$, et al: Herpesvirus saimiri-based vector biodistribution using noninvasive optical imaging. Gene Ther 2005, 12(19):1465-1476.

10. Prasad KM, et al: Robust cardiomyocyte-specific gene expression $f$ ollowing systemic injection of AAV: in vivo gene delivery follows a Poisson distribution. Gene Ther 2011, 18(1):43-52.

11. Rehemtulla A, et al: Rapid and quantitative assessment of cancer treatment response using in vivo bioluminescence imaging. Neoplasia 2000, 2(6):491-495.

12. Yang $M$, et al: Whole-body optical imaging of green fluorescent protein-expressing tumors and metastases. Proc Natl Acad Sci U S A 2000, 97(3):1206-1211.

13. Snyder $C$, et al: Complementarity of ultrasound and fluorescence imaging in an orthotopic mouse model of pancreatic cancer. BMC Cancer 2009, 9(1):106.

14. Abou-Elkacem $L$, et al: Comparison of muCT, MRI and optical reflectance imaging for assessing the growth of GFP/RFP-expressing tumors. Anticancer Res 2011, 31(9):2907-2913.

15. Smith $P G$, et al: Efficient infection and persistence of a herpesvirus saimiri-based gene delivery vector into human tumor xenografts and multicellular spheroid cultures. Cancer Gene Ther 2005, 12(3):248-256.

16. Macnab SA, et al: Herpesvirus saimiri-mediated delivery of the adenomatous polyposis coli tumour suppressor gene reduces proliferation of colorectal cancer cells. Int J Oncol 2011, 39(5):1173-1181.

17. Tomayko MM, Reynolds CP: Determination of subcutaneous tumor size in athymic (nude) mice. Cancer Chemother Pharmacol 1989, 24(3):148-154.

18. Ayers $G D$, et al: Volume of preclinical xenograft tumors is more accurately assessed by ultrasound imaging than manual caliper measurements. J Ultrasound Med 2010, 29(6):891-901.

19. Jensen $M M$, et al: Tumor volume in subcutaneous mouse xenografts measured by microCT is more accurate and reproducible than determined by $18 \mathrm{~F}-\mathrm{FDG}$-microPET or external caliper. BMC Med Imaging 2008, 8:16.

20. Keller MD, et al: Autofluorescence and diffuse reflectance spectroscopy and spectral imaging for breast surgical margin analysis. Lasers Surg Med 2010, 42(1):15-23.

doi:10.1186/1471-2342-13-35

Cite this article as: Ingram et al:: The use of high-frequency ultrasound imaging and biofluorescence for in vivo evaluation of gene therapy vectors. BMC Medical Imaging 2013 13:35.

\section{Submit your next manuscript to BioMed Central and take full advantage of:}

- Convenient online submission

- Thorough peer review

- No space constraints or color figure charges

- Immediate publication on acceptance

- Inclusion in PubMed, CAS, Scopus and Google Scholar

- Research which is freely available for redistribution 